\title{
INVESTIGACIÓN/RESEARCH
}

\section{MEDIOS DE COMUNICACIÓN, TERREMOTOS Y TSUNAMIS; LOS CASOS DE CHILE Y JAPÓN}

Rubén Gómez Quezada ${ }^{1}$ : Universidad Católica del Norte, Chile rgomez@ucn.cl

\section{RESUMEN}

El 2010 y el 2011 serán años muy difíciles de olvidar en Chile y Japón. La razón, la tragedia originada por dos terremotos de enorme magnitud y dos tsunamis devastadores que causaron miles de muertos, desaparecidos, daños colosales y lo más terrible, un ambiente de desesperanza política y cívica, desconfianza, incertidumbre y dudas sobre el manejo comunicacional en ambas catástrofes.

En este ensayo se recuerdan los hechos, se advierten las principales falencias en el manejo comunicacional y control de la información y se intenta reflexionar sobre qué podemos hacer para enfrentar de mejor manera estas catástrofes de la naturaleza. Fukushima y Chernobyl, terremotos, tsunamis y cambio climático, nos indican que es tiempo de cambiar los paradigmas en el manejo de las crisis comunicacionales. Es tiempo y necesidad de un esfuerzo nuevo, generoso, informado y participativo por superar las dificultades.

PALABRAS CLAVE: incertidumbre - manejo comunicacional - alerta temprana desastre nuclear.

\section{COMMUNICATION MEDIA, EARTHQUAKES AND TSUNAMIS: THE CASES OF CHILE AND JAPAN}

\footnotetext{
${ }^{1}$ Autor Correspondiente:

Rubén Gómez Quezada: Académico Escuela de Periodismo de la Universidad Católica del Norte, Antofagasta, Chile.

Correo: rgomez@ucn.cl.
} 


\section{ABSTRACT}

The years 2010 and 2011 will be difficult to forget in Chile as well as in Japan. The reason is, the tragedy brought about by two great earthquakes and two devastating tsunamies caused thousand deads, disappeareds, huge damages and most awfully, an invironment of political and civic hopelessness, mistrust, uncertainty and doubts on the communicative management in both catastrophes.

In this essay facts are remembered, the main mistatements are warned on the communicational management, information control together with a reflection on what we can do to cope with these nature catastrophes in a better way. Fukushima and Chernobeil, earthquakes, tsunamis and the climatic change signal that it is time to change the paradigms in the management of communicational crisis. It is also time and need of a new effort, generous, informed and joint acting to overcome the difficulties.

KEY WORDS: uncertainty - communicational management - early alert - nuclear disaster.

\section{INTRODUCCIÓN}

El 27 febrero del año pasado, un terremoto grado 8,8 en la escala Richter, azotó la zona centro sur de Chile en lo que se considera uno de los seis terremotos más fuertes y destructivos medidos instrumentalmente en el mundo. El sismo provocó un desastre de dimensiones gigantescas, aterrador. Todo ocurrió de noche, a oscuras. 30 minutos después, numerosas zonas costeras experimentaron varios maremotos, conocidos como tsunamis, lo que aumentó la destrucción, la muerte y el pánico. El epicentro se ubicó en la provincia de Ñuble, Región del Bío Bío, bajo el mar a doce kilómetros de la costa de Cobquecura y su fuerza se sintió en gran parte de Sudamérica. Seis regiones chilenas fueron afectadas, zona donde se concentra el 80 por ciento de la población nacional.

En esos días, el gobierno de la entonces Presidenta Michelle Bachelet preparaba la entrega del mando a Sebastián Piñera, lo que se concretó el 11 de marzo, traspaso que no estuvo exento de dificultades. Este, estuvo cruzado por acusaciones de mal manejo de la crisis y lo peor, una judicialización de la tragedia que aún hoy nos mantiene en ascuas, temblando bajo las réplicas. En la fatídica noche del día $27 \mathrm{~F}$, se cortó gran parte de las comunicaciones viales y de telecomunicaciones y además de la oscuridad, las zonas afectadas quedaron en un caos o en silencio absoluto, salvo los ayes de dolor, que no permitieron reaccionar ante las olas asesinas del tsunami.

Según informaciones científicas, el terremoto del $27 \mathrm{~F}$ de Chile fue 31 veces más fuerte y liberó 178 veces más energía que el que afectó a Haití el 12 de enero 2010 y 
que provocó al menos 300.000 muertos. En el terremoto chileno se calcula que la energía liberada equivaldría a unas 100.000 bombas atómicas como las lanzadas sobre Hiroshima y Nagasaki en 1945. El terremoto chileno cambió el eje de la Tierra y los días se hicieron más cortos. Informaciones satelitales y de GPS constataron que por ejemplo, la ciudad de Concepción, a unos 160 kilómetros del epicentro, se desplazó tres metros hacia el oeste; Valparaíso lo hizo unos 28 centímetros y Santiago de Chile unos 24 centímetros. Desde ese día Buenos Aires y Santiago de Chile están cuatro centímetros más cerca. El terremoto habría modificado el territorio en lugares tan distantes como Fortaleza, en Brasil y las Islas Malvinas. En otras palabras, cambió la morfología del planeta.

\section{JAPÓN: LA CATÁSTROFE COMPLETA}

El terrible escenario se repetiría en forma más apocalíptica a poco más de un año, esta vez, en Honshu, zona noreste de Japón. El 11 de marzo de este año en pleno día, un terremoto seguido de numerosos tsunamis golpeó la costa oriental japonesa provocando un escenario inimaginable. Este acontecimiento provocó la conjunción inédita de un terremoto grado 9.0 Richter, un tren de tsunamis que no había visto nunca Japón en más de mil años y más aún, un desastre nuclear quizás más grave que el sucedido en Chernobyl. Todo esto ocurre en un territorio pequeño, rodeado de mar, densamente poblado y altamente desarrollado donde la tecnología y la ciencia ayudaron a evitar en parte efectos aún más severos.

La tragedia de Honshu, a seis meses de ocurrida, significó alrededor de 30.000 víctimas, entre muertos y desaparecidos y daños mayores a los registrados en Chile. Según los informes conocidos, la mayor cantidad de víctimas y daños fue provocada por los tsunamis, con olas superiores a los 15 metros y con una penetración de varios kilómetros en el territorio japonés aplastando todo lo que encontraron a su paso, incluida la central nuclear de Fukushima.

\section{EL RECUERDO DE CHERNOBYL}

Cuando ocurrió el desastre de Chernobyl en la ex Unión Soviética el 26 de abril de 1986, una nube radioactiva sobrevoló Europa y provocó el desplazamiento de casi medio millón de personas. Actualmente, los daños por malformaciones genéticas, cánceres y radiación son de proporciones gigantescas y lo peor, no son plenamente conocidos. El martes 27 de abril pasado, justo 25 años después de ese desastre, se difundieron algunos datos aterradores. En efecto, se calcula que hoy en Bielorusia, Ucrania y la Rusia europea, hay aproximadamente unos 13 millones de personas que siguen afectadas por la radioactividad persistente en la zona. Peor aún; en Bielorusia se calcula que sólo el 20 por ciento de los niños pueden ser considerados sanos y que el daño cerebral es la peor consecuencia de la catástrofe, seguida de diversos tipos de cánceres. El doctor de la Universidad de Wisconsin, J effery Patterson explicó hace poco: "no sabemos absolutamente nada de los efectos genéticos que la radiación pueda causar en el hombre y tendremos que observar a generaciones y generaciones 
de afectados, por cientos de años, para llegar a conocer los efectos reales del desastre nuclear de Chernobyl".

Desde el punto de vista comunicacional, lo más grave, sin embargo, es la manera de exponer los hechos. Algunos sostienen que en 1986 en el momento de la explosión del reactor nuclear soviético, fallecieron directamente 50 liquidadores (militares y civiles que sacrifican su vida para controlar la tragedia in situ). En tanto, para la Organización Mundial de la Salud OMS, y la Agencia Internacional de Energía Atómica, AIEA, las víctimas indirectas de la tragedia no habrían superado las 9.000 almas. Otras fuentes no gubernamentales y de la sociedad civil, argumentan que la OMS y la AIEA mintieron groseramente y sitúan las víctimas fatales entre abril de 1986 y diciembre del 2004 en 985.000 personas. Es decir, casi un millón de muertos por el accidente nuclear.

Volviendo al presente año, la tragedia japonesa revivió los peores fantasmas. Durante un mes, en la central Fukushima, se ocultó información sobre la gravedad de los daños. Las razones pueden ir desde cálculos económicos, incapacidad técnica para evaluar los daños o simplemente mala intención. Se partió señalando grado 3, luego grado 4 y así sucesivamente, un mes después se alcanzó el grado 7, el máximo de la escala y que correspondió en su oportunidad a Chernobyl, en Ucrania. Más grave aún, durante varios días no se informó de accidente. Sólo después se comenzó a ordenar la evacuación de las áreas cercanas cuyos residentes no podrán volver a casa durante varias décadas. Durante semanas, lo que se reconoció era un accidente localizado, se comprobó estaba fuera de control y alcanzó el agua del mar, el agua potable de Tokio y otras ciudades y provocó el pánico, el éxodo y la estampida de muchos extranjeros desde el Japón. Cosa inédita, provocó también la desconfianza de la población japonesa contra la empresa eléctrica privada que maneja la energía nuclear, en este caso Tepco y luego, del gobierno japonés. La situación es aún más incierta por cuanto Japón, se abastece casi en un 25 por ciento de energía a partir de los 54 reactores nucleares ubicados en su territorio los que quedaron paralizados con la catástrofe.

Lo terrible es que la realidad superó las peores pesadillas. Algunos expertos sostienen que pese a las lecciones de Chernobyl, 25 años después en Japón, el gobierno utilizó las mismas recetas para hacer frente al desastre. Es decir, hubo secretismo, ocultamiento de la información y minimización de las consecuencias de los hechos ocurridos.

Cabe señalar que a nivel mundial existen hoy unos 440 reactores nucleares en actividad. Francia, es el país con más energía nuclear ya que abastece con ella alrededor del 76 por ciento de sus necesidades energéticas. En cuanto a nuestro continente, bien sabemos Argentina, Brasil y México tienen dos centrales nucleares cada uno. En el caso brasileño, se trata de Angra 1 y 2 en la zona costera de Angra dos Reis, en el estado de Rio de Janeiro y a nivel nacional abastece con ellas sólo el 3 por ciento de sus necesidades.

\section{COMUNICACIONES Y CATÁSTROFES}


Los terremotos de Chile y Japón, así como los desastres nucleares de Chernobyl y ahora Fukushima, ponen de relieve la importancia que tiene el manejo de las comunicaciones para prevenir y afrontar adecuadamente las catástrofes naturales y de accidentes tan graves como los nucleares. No es un tema menor, sobre todo si constatamos que hoy el planeta experimenta un cambio climático con consecuencias imprevisibles.

En el caso chileno, una revisión de los principales medios de comunicación, así como los trabajos de opinión en diarios durante parte del 2010 y del 2011, permite sacar algunas conclusiones preliminares. Entre ellas, el tipo de cobertura de las informaciones es de naturaleza reactiva. Es decir, se publica abundante información luego de ocurridos los desastres y se pudo comprobar que la magnitud del terremoto del $27 \mathrm{~F}$ y el posterior tren de tsunamis, provocó durante horas confusión e incluso colapso de los sistemas públicos y gubernamentales para enfrentar la catástrofe. Afortunadamente, una norma sísmica de muchos años, permitió que pese a los ingentes daños en infraestructura, el país no sucumbiera en su totalidad. Pero, todo indica, que el manejo de la crisis fue tan o más grave que la misma emergencia.

Los medios de comunicación chilenos publicaron las repercusiones del desastre y acudieron en la mayoría de los casos a la opinión de expertos, lo que se acompañó siempre de testimonios de víctimas, los que compitieron en innumerables ocasiones a tratamientos de espectacularidad creciente e incluso, de grados de morbosidad innecesarios, pero no se caracterizaron por diseñar y practicar anticipadamente, salvo contadas excepciones, campañas sistemáticas masivas, permanentes, de cómo prevenir adecuadamente y cómo enfrentar situaciones límites.

La revisión de lo publicado, permite configurar la idea de que prácticamente el país quedó sin comunicación vial, de telecomunicaciones y sin control de daños, es decir, sin un aparato estatal que pudiera leer lo que estaba pasando en el territorio nacional durante varias horas. No hubo comunicación efectiva entre la Oficina Nacional de Emergencia, Onemi y la Armada lo que impidió dar la alarma de tsunami a la población durante seis o siete horas, pese a que estos comenzaron 34 minutos después del terremoto. Estas fallas son absolutamente impresentables desde el punto de vista de la eficiencia en el manejo de información pública, de crisis, que es un derecho de los ciudadanos.

Más complicado aún, el Centro de Alertas de Tsunamis del Pacífico a pocos minutos del terremoto dio una alarma generalizada a la cuenca del Océano Pacífico que incluyó más de cincuenta países. Pero nada de esa información funcionó en Chile. Sin duda, desde el punto de vista comunicacional, esto es muy grave y amerita un esfuerzo periodístico por tratar de encontrar algunas explicaciones razonables ¿Qué pudo haber pasado y qué puede hacerse para disminuir a futuro los efectos de estos escenarios tan catastróficos?

En este esfuerzo pueden ensayarse algunas explicaciones. La primera sería que la magnitud de la tragedia fue tal que el sistema de prevención, alerta y seguimiento del 
Estado país no funcionó y básicamente por su excesiva dependencia del centro del territorio, las insuficiencias técnicas, científicas, administrativas y de recursos disponibles y por un diseño inadecuado de las estructuras del manejo de crisis que debe estar integrado por expertos en comunicación y en particular también por periodistas y cientistas sociales, entrenados, motivados y calificados.

Lo segundo, sería que nuestra reacción en tanto que periodistas, comunicadores, publicistas, propagandistas, etc, es básicamente reactiva y no guarda relación con la anticipación desde una estructura fuerte del Estado que permita alianzas anticipadas con los medios de comunicación y los sectores productivos privados. El deber primario de cuidar a la población no es de los periodistas y comunicadores, sí de un Estado claro en sus compromisos con la sociedad.

Tercero; en Chile pese a la reiteración de fenómenos telúricos y de tsunamis, hay insuficiente información científica que permita comprender exactamente lo que está pasando. Por tanto, reducir los efectos en la búsqueda de responsabilidades políticas es achicar la comprensión del fenómeno y su importancia. En ese sentido, el hecho que el terremoto y sus efectos haya entrado en una etapa de judicialización a la cual responderán en su momento las principales autoridades del Estado chileno, sean estas autoridades políticas, militares o de orden, no parece ser lo más adecuado.

Podríamos preguntarnos si esta judicialización o politización del tema aportará en concreto a disminuir los efectos de catástrofes similares. Consuelo menor, en Japón este año, el terremoto y posterior tsunami afectó al país más desarrollado del mundo en materia de prevención, defensas y alertas tempranas y que cuenta con la más completa organización cívica y de mitigación de desastres. Sin embargo, los muertos alcanzaron casi las 30.000 personas y los daños multiplicaron por muchas veces los ocurridos en Chile.

Cuarto, esto significa que nada garantiza salir ilesos de una catástrofe de estas proporciones, menos en países donde las posibilidades de terremotos son permanentes. Se necesitan más científicos, más expertos, más inversión en prevención, más equipamiento, más ejercicios de evacuación y señaléticas, más periodistas y comunicadores que manejen las crisis y más escrutinio público. Es decir, se necesitan Estados nacionales más fuertes y medios de comunicación disponibles para sacrificar ganancias económicas en pos del interés común.

\section{REVISANDO PARADIGMAS}

Estos hechos y catástrofes forzosamente están cambiando varios paradigmas. El científico Edwin Lyman desde Washington, citado por el periodista chileno Juan Manuel Vial en el diario La Tercera se refiere al tema de los mitos pre-Fukushima: Esto es más menos lo que se daba como verdad absoluta: "imposible que vuelva a suceder algo como Chernobyl. La nueva generación de plantas nucleares es más segura; la basura nuclear puede ser reciclada" Lyman añade ahora lo que denomina los mitos post desastre japonés: "algo como Fukushima jamás podría suceder en Estados Unidos; Fukushima no fue tan terrible como Chernoby; la próxima generación 
de plantas nucleares sí serán más seguras". Datos nuevos; un temblor 5,8 grados Richter afectó el mes pasado (agosto) parte de la costa este de Estados Unidos y provocó al menos el cierre de dos centrales nucleares en Virginia, desconociéndose en detalle otros problemas eventuales. Otras plantas nucleares cerraron su actividad en la costa este hace pocos días tras el paso del huracán I rene.

¿Qué hay de vasos comunicantes en estas últimas tragedias tanto en Chile, Japón como en Chernobyl hace 25 años y que demostraron claramente un mal manejo comunicacional de la crisis y la necesidad de un esfuerzo nuevo, generoso, informado y participativo por superar las falencias?

Las respuestas pueden ser variadas, pero hay algunas que quizás expliquen o hagan más entendible el problema y su solución:

\section{EL CASO CHILENO:}

a) Chile tiene una tradición de terremotos lo que supone por ejemplo que en materia de construcción, las normas antisísmicas son mejores que en otras latitudes, aunque evidentemente no son perfectas. Balance del $27 \mathrm{~F} 2010$ : alrededor de 550 muertos. Unos 160 corresponderían a efectos de los tsunamis. 800.000 damnificados y daños estimados en unos 30.000 millones de dólares junto con un descalabro de la industria aseguradora.

b) Según científicos chilenos encabezados por el sismólogo Raúl Madariaga, en realidad fueron dos terremotos los que sacudieron la zona sur del país el $27 \mathrm{~F}$. El primero y que duró dos minutos y 45 segundos, alcanzó 8,8 grados y el segundo, casi en forma inmediata, se localizó más al norte, entre Constitución y Pichilemu por la costa. Es decir hubo dos zonas de liberación gigantesca de energía en una extensión de más de 500 kilómetros. Es decir, ambos megasismos atacaron el "hinterland" chileno. Precisamente, el terremoto más pequeño habría alcanzado 8,4 grados Richter y habría ocasionado no obstante, los mayores daños al amplificar los efectos del terremoto mayor y las ondas de trenes de olas gigantescas que tomaron cursos insospechados.

c) La realidad superó todos los planes previos. El sismo ocurrió de madrugada, y provocó un apagón de la electricidad en todas las regiones amagadas, lo que imposibilitó la comunicación entre el gobierno (en transición por cambio de mando) y la Oficina Nacional de Emergencia, Onemi. Evidentemente, Chile desperdició una vez más su potencial de energía solar para reemplazar la electricidad.

d) La legislación chilena prohíbe que las fuerzas armadas actúen motu propio. Y debido a que no hubo información ni comunicación durante varias horas, reinó el caos, la inoperancia y la desorientación absoluta. La Onemi y la Armada todavía se recriminan mutuamente que no se haya dado una alarma de tsunani e incluso que cuando se dio la misma, en forma quizás poco clara, se la levantó pese a que los maremotos persistieron durante varias horas ininterrumpidamente en las sombras.

e) El Estado chileno estaba preparado en el papel para un terremoto previsible, casi como para una respuesta de manual. Lo que pasó la madrugada del $27 \mathrm{~F}$ 
fue que imperó la sorpresa total, el desconcierto absoluto. Al no existir información, ni comunicación, no hubo manejo adecuado de la crisis. Se esperaba un desastre y una catástrofe limitada y no fue así; se esperaba un incremento pausado de daños y efectos y no fue así, fue brutalmente impredecible. Se esperaba una catástrofe de desarrollo lento y no fue así. La catástrofe fue instantánea, integral y de una potencia descomunal.

f) Por último, sucedió a menos de dos semanas del cambio de mando presidencial, lo que acrecentó una cierta sensación de vacío de poder.

\section{EN EL CASO J APONÉS}

a) El terremoto japonés alcanzó 9,0 grados Richter, el quinto más potente de la historia y provocó tsunamis de características brutales. Consecuencia: alrededor de 30.000 muertos y desaparecidos; daños aproximados a los 235 mil millones de dólares. Unas diez veces más que en Chile. La mayor parte de las víctimas corresponde a los daños del tsunami.

b) Japón, además de estas dos tragedias sufrió la catástrofe nuclear más grande de la historia. En Fukushima, el tsunami dañó tres de los seis reactores de su planta nuclear provocando una catástrofe de dimensiones inciertas. Hasta varios meses de ocurrida la catástrofe aún no se puede ingresar a la planta nuclear ni normalizar la situación. Existe una zona de exclusión de más de 30 kilómetros a la redonda donde no puede ingresar persona alguna. Peor aún, toda actividad humana y productiva en todo ese perímetro y sus adyacencias está prohibido por tiempo indefinido.

c) El manejo comunicacional fue similar al adoptado en Chernobyl. Primero se mantuvo el desastre en secreto, luego, se ocultó la información más sensible y por último, se minimizó sus efectos. La emergencia aún no ha sido superada y en la zona de Fukushima desapareció la vida y aún crece el riesgo de radioactividad y el pánico. La aparente tranquilidad y espíritu cívico aguantó un tiempo. Meses después de la tragedia las encuestas, los expertos y las imágenes de televisión y la prensa dan cuenta que los japoneses no creen ni confían en sus autoridades políticas, ni en la empresa privada Tepco que maneja la central. La crisis de desconfianza causó la renuncia hace pocas semanas del Primer Ministro y es quizás el efecto menos esperado de esta trilogía trágica.

d) Japón antes del terremoto del 11 de marzo era considerado como el país más avanzado en materia de prevención, defensa y educación para las catástrofes. Además, contaba con la mayor red de vigilancia de sismos y un sistema de alerta temprana de tsunamis. Pues bien, la catástrofe demostró que ninguna tecnología puede evitar las tragedias de la naturaleza, aunque sí puede mitigar en parte los daños.

\section{MEDIOS, SENTIDO COMÚN Y CATÁSTROFES}

Como sabemos, los medios de comunicación obedecen fundamentalmente a las reglas del mercado y consideran a la noticia como una mercancía. No extraña entonces que luego de producidas las catástrofes se realicen completas coberturas con los efectos 
de las mismas. Cunde la información ligada a la exacerbación de los efectos de la tragedia, la morbosidad y los elementos ligados a la rareza, el conflicto, el suspenso y la trama dramática.

Según coinciden expertos, los medios de comunicación no siempre tienen protocolos específicos de cómo enfrentar las catástrofes, menos aún de cómo prevenirlas, no es parte de su esencia del día a día. Por la naturaleza de estos medios en el caso de que existieran políticas de prevención, de difusión y similares, estas en el mejor de los casos son genéricas y no consideran las particularidades de una población heterogénea y en condiciones de riesgo tan disímiles como inabarcables. La poca información disponible, generalmente se realiza a través de insertos pagados y suplementos especiales o bien son reactivos a la ocurrencia de eventos catastróficos. La información específica que podría considerarse técnica por lo general está situada a nivel de manuales, guías de acción o campañas esporádicas de organismos gubernamentales, los que muchas veces pecan de falta de actualización o de profundización y peor aún, son centralizadas a nivel país, invisibilizando las realidades regionales y locales.

\section{EL EJEMPLO DEL NORTE CHILENO}

Cabe destacar que en el norte de Chile, hay una práctica más habitual de ejercicios de prevención de terremotos, tsunamis y otros. Esto se debe en gran parte a la certeza que en algún momento debería producirse un sismo de grandes dimensiones y eventuales tsunamis en la zona comprendida entre Arica y Antofagasta. Coincidentemente, en esta zona se ubican también las mayores empresas mineras del país, tanto privadas como estatales las que están desarrollando políticas de colaboración con organismos técnicos y de gobierno, lo que es un avance importante sobre todo después de los acontecimientos del $27 \mathrm{~F}$. Más ilustrativo es el hecho de que en el terremoto de Japón de 2011, se decretó una alerta de tsunami en el Océano Pacífico y que incluyó toda la costa chilena. En la ocasión, se realizaron medidas masivas de evacuación de zonas costeras de riesgo en una maniobra políticamente peligrosa pero acertada desde el punto de vista de la seguridad.

El viernes 19 de agosto recién pasado, la Región de Antofagasta realizó el mayor simulacro de terremoto y tsunami de la historia de Chile. El ejercicio realizado en la zona costera y el interior contó con la participación de unas 400.000 personas. En la ciudad de Antofagasta, en menos de 20 minutos, el ulular de las sirenas anunció un terremoto simulado de 8,3 grados Richter lo que llevó a la evacuación hacia las zonas de seguridad a unas 135 mil personas, incluidos los alumnos de colegios, clínicas de salud, hospitales, comercios, oficinas públicas, y domicilios. En el interior, más del 80 por ciento de la población aplicó los protocolos de seguridad alejándose de vidrios y buscando refugio en los lugares predeterminados. Para el 2012 se hará un nuevo ejercicio y no se conocerá ni el día ni la hora y se espera contar además con un sistema nacional de alerta temprana en televisores y teléfonos celulares. Sin duda, este tipo de ejercicios es de enorme importancia ya que familiariza a la población con el riesgo y se pueden estudiar medidas para mitigar los daños. 
Insistiendo en el tratamiento de los temas de manejo de crisis por catástrofes en los medios de comunicación, rara vez se encuentran artículos referidos a la prevención y autocuidado. La excepción, quizás está señalado, como se indicó anteriormente, en la prensa del norte del país que tiene una mayor conciencia sobre el tema. En el simulacro gigante del mes pasado en Antofagasta, de los tres canales de TV por cable de la ciudad, dos de ellos transmitieron en directo y durante los 45 minutos del ejercicio todas las alternativas del mismo. Un tercero, sólo se dedicó a programas de entretención. Las radios locales prácticamente todas participaron del ejercicio. Ningún canal de televisión de alcance nacional transmitió en directo el acontecimiento relegando la información a noticieros o ediciones de horarios o días posteriores.

Paralelamente, una revisión acuciosa de los espacios de opinión de los principales diarios chilenos y de los temarios de sus principales columnistas y comentaristas después del $27 \mathrm{~F}$ chileno y hasta mediados de este año, demostró que el tema del manejo de la información en catástrofes como las vividas o la relevancia del manejo de crisis es prácticamente nulo. No es tema. En la idea que los medios de comunicación de masas son instrumentos que permiten la formación de la opinión pública y por ende de las conductas y hábitos de los ciudadanos y de su cultura cívica, sin duda existen falencias considerables.

De allí entonces que urge una mirada distinta al problema y que debe ser encarada de preferencia por las universidades e investigadores y mediadores comunicacionales que son los periodistas y agentes de producción de noticias, y responsables de campañas publicitarias, propagandísticas y educadores. Obviamente que hay una carencia que debe ser trabajada seriamente a través de las facultades de comunicación y periodismo, de campañas educativas, formativas, y donde converjan las tecnologías para la prevención y manejo de las crisis anteponiéndose siempre el interés general y colectivo. Esto implica diseñar estructuras descentralizadas, poderosas, competentes que puedan articular experiencias, aportes, y comunión de la mayor cantidad de actores de la sociedad local.

\section{UN TERRENO ABONADO}

En el caso específico de nuestra facultad, en la Universidad Católica del Norte, ubicada en una zona de riesgos de terremotos y tsunamis de primera importancia, nuestra escuela de periodismo ha desarrollado un trabajo de largo aliento, muchas veces incomprendido pero que adquiere cada vez más importancia. Qué duda cabe que ante un escenario absolutamente imprevisible debido, entre otros, al cambio climático y sus consecuencias insospechadas por lo extenso de sus daños y repercusiones, y nuestras características geológicas, el manejo de las comunicaciones, en particular las comunicaciones de crisis de cualquier naturaleza, pero con énfasis en terremotos, tsunamis y tragedias es absolutamente vital y debe ser parte consistente del curriculum de comunicadores periodistas en asociación con expertos y científicos, organismos públicos y privados y necesariamente la comunidad organizada. Esta es una tarea que se ha emprendido en nuestra escuela silenciosamente durante varios 
años a través de cátedras, talleres, congresos y seminarios y que seguramente será parte integral de cursos de profundización y especialidades de post grado desde el punto de vista de las comunicaciones y el periodismo.

Pero queda todavía una gran tarea pendiente. Actualmente, en Chile la educación cívica está muy debilitada. Y ello se debe en gran medida a que el relato imperante en las últimas décadas ha minimizado el valor de las ciencias sociales en la suerte de los habitantes y el colectivo país. Esto puede manifestarse por ejemplo en la falta de una complementación educativa en materia de catástrofes y en el manejo de crisis. Faltan también medios de comunicación estatales que tengan autonomía o fórmulas mixtas para instruir, enseñar, prevenir y liderar procesos donde la información o su ausencia signifique la diferencia entre la vida y la muerte. La figura del funcionario público, del que adopta decisiones políticas, ha sido vilipendiada hasta el cansancio y ello es también una situación grave que erosiona los niveles de credibilidad en el momento de ocurrencia de tragedias en las cuales hay que confiar en el que gobierna y en aquellos en que el gobierno delega por competencias y habilidades técnicas, la responsabilidad de tomar las mejores decisiones ante emergencias.

\section{A MODO DE CONCLUSIÓN}

Como lo demostró Japón, es imposible evitar los daños de un terremoto, un tsunami o una catástrofe nuclear. Lo que sí se puede hacer es morigerar sus consecuencias. Para ello, además de la educación, se requieren recursos y un Estado fuerte, generoso y proactivo que oriente, y conduzca la prevención y el manejo de las crisis.

Lo ideal es entender que hay que ejercitarse ante el peor escenario posible, para lo cual un Estado responsable no debería escatimar en recursos. Nada de lo anterior sirve si no se realizan sostenidamente ejercicios y simulacros que logren concitar el apoyo de los sectores público y privado y la comunidad organizada.

La solidaridad y el sentido común son piezas claves. Se mantiene la máxima de que cuando no puedes mantenerte en pie por la fuerza de un sismo y estás cerca de la costa, la idea es que apenas cese el movimiento hay que escapar a los lugares más altos, sin esperar ni siquiera las sirenas. Pero, no puedes escapar en automóviles, ocupando las vías de evacuación de ambulancias. Hay que escapar a pie hacia las zonas altas. Pero, aquellos que no pueden hacerlo por sus propios medios deben ser evacuados por los organismos del Estado o los vecinos. Si no hay solidaridad, sin duda vendrá el caos. Aunque parezca trivial, si no hay señaléticas entendibles y socializadas, no hay comprensión de los fenómenos. Si nuestras ciudades y pueblos no tienen diseños viales, ni planos reguladores inteligentes, seguramente será una tragedia de proporciones.

Las fuerzas armadas y de orden no pueden permanecer impávidas esperando instrucciones que quizás nunca lleguen. En esos casos deberían actuar de inmediato para socorrer a los más necesitados.

Lo más complicado es entender que sin comunicación oportuna y yo agregaría sentido 
común, no se pueden tomar decisiones. En el caso de catástrofes naturales, la información y la comunicación son vitales para que haya decisiones políticas y técnicas adecuadas. De allí entonces que se requieren periodistas y profesionales de la comunicación empoderados de las herramientas adecuadas y que no actúen sólo por reacción o en busca del rating o de lo morboso.

La necesidad de contar con alarmas tempranas es también imperiosa, así como el uso y manejo de información científica de calidad que permita disminuir riesgos ante catástrofes. Ello requiere recursos económicos muy grandes que deben ser entregados en función del interés público. Como muestra de lo que no puede pasar es que el 27 $\mathrm{F}$ en Chile no hubo disponibilidad de teléfonos satelitales y no hubo uso del potencial de energía solar para producir electricidad. La necesidad de una cultura permanente del simulacro en los escenarios más duros y potencialmente más dañinos, es también una herramienta a privilegiar. Debe haber una adecuada formación, manejo y transmisión de las alarmas, instrucciones y roles en un ambiente fluido, despolitizado y técnico político responsable en el cual los habitantes del país y los ciudadanos puedan confiar. Esta es una tarea urgente que va más allá de los partidos políticos o grupos económicos y sociales y es una oportunidad de descubrir o redescubrir las inmensas posibilidades de colaboración en sociedades y comunidades abiertas, integradas y preocupadas colectivamente de su destino y su calidad de vida.

\section{Rubén Gómez Quezada}

Rubén Gómez Quezada, profesor de la Escuela de Periodismo de la Universidad Católica del Norte, Antofagasta, Chile. Director de la Revista de Comunicaciones, Periodismo y Ciencias Sociales Tercer Milenio (http://tercermilenio.periodismoucn.cl) Magister en Comunicación Social por la Pontificia Universidad Católica de Chile con mención en Edición y Dirección Periodística. Dicta cursos de periodismo de interpretación, opinión y narrativa periodística. Realiza investigación en Historia y Medios de Comunicación. Coautor del libro 50 años de la Historia de Chile: Vistos a través de la Revista Mensaje Tomo I (2008); Tomo II (2011) 